\title{
Determination of value of bovine respiratory disease control using a remote early disease identification system compared with conventional methods of metaphylaxis and visual observations ${ }^{1}$
}

\author{
B. J. White, ${ }^{*} \dagger^{2}$ D. E. Amrine, * and D. R. Goehl* \\ *Precision Animal Solutions, LLC, 210 Southwind Rd., Suite 204, Manhattan KS 66503; \\ and $\dagger$ Kansas State University, Dept. of Clinical Sciences, Manhattan 66506
}

\begin{abstract}
Mitigation of the deleterious effects of bovine respiratory disease (BRD) is an important issue in the cattle industry. Conventional management of calves at high risk for BRD often includes mass treatment with antimicrobials at arrival followed by visual observation for individual clinical cases. These methods have proven effective; however, control program efficacy is influenced by the accuracy of visual observation. A remote early disease identification (REDI) system has been described that monitors cattle behavior to identify potential BRD cases. The objective of this research was to compare health and performance outcomes using either traditional BRD control (visual observation and metaphylaxis) or REDI during a 60-d postarrival phase in high-risk beef calves. The randomized controlled clinical trial was performed in 8 replicates at 3 different facilities over a 19-mo period. In each replicate, a single load of calves was randomly allocated to receive either conventional management (CONV; total $n=8$ ) or REDI (total $n=8$ ) as the meth-
\end{abstract}

od for BRD control. Cattle were monitored with each diagnostic method for the first $30 \mathrm{~d}$ on feed and performance variables were collected until approximately $60 \mathrm{~d}$ after arrival. Statistical differences $(P<0.10)$ were not identified in common performance (ADG) or health (morbidity, first treatment success, and mortality risk) among the treatment groups. Calves in the REDI pens had a lower $(P<0.01)$ average number of days on feed at first treatment $(9.1 \pm 1.2 \mathrm{~d})$ compared with CONV pens $(15.8 \pm 1.2 \mathrm{~d})$. There were no statistical differences $(P>0.10)$ in risk of BRD treatment and REDI calves were not administered antimicrobials at arrival; therefore, REDI calves had a lower $(P<0.01)$ average number of doses of antimicrobials/calf $(0.75 \pm$ 0.1 doses) compared with CONV calves $(1.67 \pm 0.1$ doses). In this trial, the REDI system was comparable to conventional management with the potential advantages of earlier BRD diagnosis and decreased use of antimicrobials. Further research should be performed to evaluate the longer-term impacts of the 2 systems.

Key words: behavior, bovine respiratory disease, bovine respiratory disease diagnosis (C) 2015 American Society of Animal Science. All rights reserved. $\begin{array}{r}\text { J. Anim. Sci. 2015.93:4115-4122 } \\ \text { doi:10.2527/jas2015-9079 }\end{array}$

\section{INTRODUCTION}

Bovine respiratory disease (BRD) is common in postweaned beef calves (Smith, 2009; USDA, 2013), and deleterious consequences of BRD result in loss-

\footnotetext{
${ }^{1}$ Funding for this project was provided in part through Merck Animal Health, Summit, NJ, and Professional Beef Services, Canton, MO. Precision Animal Solutions, LLC, holds all intellectual property rights (patent pending) for the remote early disease identification (REDI) system.

${ }^{2}$ Corresponding author: bradwhite@probeef.net

Received March 7, 2015.

Accepted June 11, 2015.
}

es of up to US\$291/animal with individual animal net returns decreased based on the number of times each calf is treated (Fulton et al., 2002; Brooks et al., 2011; Cernicchiaro et al., 2013). Identifying effective methods to accurately diagnose and control BRD is important to the industry.

Although visual observation is the most common method for identifying BRD cases, diagnosis based solely on clinical signs has relatively low sensitivity and specificity (White and Renter, 2009). Cattle behavior can also be monitored remotely and may be indicative of changes in wellness status (Theurer et al., 2013a). Previous work documented changes in feeding patterns during naturally occurring BRD 
(Wolfger et al., 2015b) and calf activity following induced Mannheimia pneumonia (Hanzlicek et al., 2010; White et al., 2012; Theurer et al., 2013b). Some information exists evaluating potential economic efficiency of remote monitoring to diagnose BRD (Wolfger et al., 2015a), yet little work exists comparing remote monitoring systems to conventional BRD control mechanisms including visual observation and metaphylaxis.

The research objective was to determine potential health and performance differences between a remote early disease identification (REDI) system and a conventional BRD management system consisting of visual observation combined with metaphylaxis. The hypothesis was that cattle conventionally managed may have fewer initial cases of BRD, but fewer total doses of antimicrobial agents would be used in the pens where the REDI system was used.

\section{MATERIALS AND METHODS}

All procedures were conducted in accordance with a protocol approved by the Professional Beef Services, LLC, Institutional Animal Care and Use Committee.

The study was a randomized controlled clinical trial conducted in 8 replicates at 3 locations to compare 2 BRD arrival management methods: cattle conventionally managed with metaphylaxis at arrival and visual observation to diagnose BRD (conventional management $[\mathbf{C O N V}]$ ) or cattle receiving no metaphylaxis with all disease identification based on REDI. In this trial, pen was the experimental unit and for each replicate, whereas crossbred beef cattle arrived in a single cohort (truckload) and were randomly allocated to a REDI or CONV pen using a random number table generated in Excel (Microsoft Corp., Redmond, WA).

All cattle were housed in outdoor open-air pens with 1 truckload split among 2 pens. The pens provided a minimum of $0.45 \mathrm{~m}$ of bunk space per animal with minimum pen size of 25 by $45 \mathrm{~m}$. Three different facilities were used to complete the research: 2 facilities in Missouri and 1 in Kansas. Grass hay was made available upon arrival and cattle were gradually transitioned to the total mixed ration used by that operation, which typically contained wet gluten, soy hulls, and ground hay. The amount of feed delivered to each pen was recorded daily. Water was available ad libitum through automatic dispensers in each pen.

A naturally occurring disease model (purchase of calves at high risk for BRD) was used to evaluate potential differences between CONV and REDI for BRD control. Cattle were purchased through an order buyer as commingled calves and typically shipped a minimum of $640 \mathrm{~km}$. Upon arrival or within $12 \mathrm{~h}$, all calves were individually identified with ear tags (Allflex
USA, Dallas-Fort Worth, TX), BW were collected, and calves were immunized for clostridial (Vision 7; Merck Animal Health, Summit, NJ) and respiratory viral pathogens (Vista 5; Merck Animal Health). Cattle assigned to the REDI group within each replicate also received REDI ear tags (MKW Electronics $\mathrm{GmbH}$, Weibern, Austria). Calves in both the REDI and CONV groups were processed after daily feeding at trial midpoint and trial conclusion to collect BW.

All cattle in the CONV group received ceftiofur crystalline free acid, $6.6 \mathrm{mg} / \mathrm{kg}$ BW (Excede; Zoetis, Florham Park, NJ) subcutaneously in the base of the ear, at arrival. Cattle in the CONV group were observed daily for clinical signs of BRD by trained personnel and a 5-d postmetaphylaxis treatment interval was observed. All cattle observers were experienced with identifying BRD cases based on typical clinical signs of depression and anorexia. After the post-metaphylaxis interval, CONV cattle deemed as exhibiting signs of BRD by the observer were taken to the chute, and if rectal temperature was elevated $\left(>40.0^{\circ} \mathrm{C}\right)$ and posttreatment eligibility requirements were met as described in the treatment protocol, the calf was treated.

All cattle in the REDI pens were monitored using a system consisting of ultrawide band tags applied to individual calves and readers positioned on the periphery of the housing unit. At application of each tag, the REDI tag was associated with the visual identification of each calf, so disease calls were conveyed to the health care providers using the visual identification system. The system collected real-time positional information on each animal, and previously created algorithms (White et al., 2015) were used to determine daily health status based on several social and behavioral indices. In the previous work, the REDI system (Precision Animal Solutions, Manhattan, KS) has illustrated fair agreement with visual observation (White et al., 2015). These REDI algorithms are used to detect changes in cattle activity (e.g., distance traveled, percent of time spent active), location (e.g., percent time near areas of interest such as water and feed), and social patterns (e.g., time spent in group and in isolation). The REDI system provided a daily health status report each morning to alert healthcare personnel as to those cattle requiring potential treatment. Calves identified as ill in the REDI pens were removed from their pen for treatment at the same time period as cattle identified as ill in the CONV pens, typically late morning.

In the first 3 replicates (May, July, and August 2013), the REDI system required $3 \mathrm{~d}$ of baseline data before making a disease call, and the visual observer made all disease determinations for all cattle for the first $3 \mathrm{~d}$ after arrival. In the subsequent 5 replicates, the REDI system required $36 \mathrm{~h}$ of baseline data and a visual observer 
made all disease identification during this time period. The change in the amount of baseline data required was based on creation of a short-term algorithm that would generate REDI calls between 36 and $72 \mathrm{~h}$ after arrival. During this baseline data collection period, any cattle deemed diseased by the observer were removed from the trial. After the baseline data period, all treatment decisions in the REDI pen were based on the daily call of their health status using the preestablished disease detection algorithms. If cattle were deemed ill by REDI and they met posttreatment eligibility requirements outlined in the treatment protocol, they were treated with the appropriate therapy based on the protocol. In the REDI treatment group, rectal temperature was recorded, but it did not influence the treatment decision.

The first time a calf was determined to require BRD treatment (CONV or REDI), tildipirosin (Zuprevo; Merck Animal Health) was administered subcutaneously in the neck at the labeled dose of $4 \mathrm{mg} / \mathrm{kg} \mathrm{BW}$. Cattle were not eligible for retreatment for $5 \mathrm{~d}$ past the initial treatment; if they were deemed sick, they were not treated during this posttreatment moratorium. If cattle were identified as ill after this interval, they were administered florfenicol and flunixin (Resflor; Merck Animal Health) via a subcutaneous injection in the neck at the labeled dose of $40 \mathrm{mg}$ florfenicol/ $\mathrm{kg} \mathrm{BW}$ and $2.2 \mathrm{mg}$ flunixin/ $\mathrm{kg} \mathrm{BW}$ and were not eligible for treatment for an additional $5 \mathrm{~d}$ after this injection. If cattle were deemed ill a third time, they were administered oxytetracycline (LA300; Norbrook Laboratories, Lenexa, KS) subcutaneously in the neck at the labeled dose. As REDI was used only to identify BRD cases, pen riders observed both REDI and CONV pens for additional health disorders (e.g., lameness, pinkeye, digestive, and neurological conditions). If disease other than BRD was identified, cattle were treated according to standard treatment protocol of the operation.

\section{Statistical Analysis}

Management systems for BRD were administered at the pen level; therefore, analyses were conducted using pen as the experimental unit. Data were summarized for each pen within each replicate and imported into statistical software ( $\mathrm{R}$ version 3.0.2; R Core Team, Vienna, Austria) for analyses. Generalized linear models were used to evaluate potential relationships among continuous variables (ADG, days to first treatment, average doses of antimicrobials per calf, and rectal temperature at BRD treatment) and treatment group while accounting for a lack of independence among treatment groups due to replicate and study site. Generalized linear models were used to evaluate individual animal data to potential associations between ADG (to mid- point and trial end) and treatment group, the maximum number of times a calf was treated, and the potential interaction between these variables. Generalized mixed models were used to model proportions (morbidity, retreatment, and death risk) and potential associations with treatment while accounting for hierarchy within replicate and study site. Associations with $P$-value $\leq$ 0.05 were considered significant. A Cox proportional hazards model with frailty for replicate within site was used to evaluate the potential differences in timing of first treatment for BRD among REDI and CONV pens.

\section{RESULTS}

The replicates arrived in 8 cohorts over a period of 19 mo between May 2013 and November 2014 (Table 1). There were no differences $(P \geq 0.28)$ in initial BW or the number of calves among treatment groups by replicate (Table 2). The REDI system required baseline data prior to making BRD calls and calves deemed ill in the first 72 (replicates 1-3) or $48 \mathrm{~h}$ (replicates 4-8) were removed from the study. This resulted in removal of 3, 2, 1, 1, and 2 calves from replicates 1,2, 3, 4, and 5 respectively. No calves were removed prior to REDI calls in replicates 6,7 , or 8 .

Midpoint weights were recorded between d 30 and 33 and each trial lasted between 53 and $67 \mathrm{~d}$. Logistical issues forced a premature endpoint to replicate 6 (Kansas location) and all data collection ceased after the midpoint weight as the cattle were sold. Data from this location are included only to the midpoint.

No differences were identified $(P>0.10)$ in health (number of first treatments, first treatment success, or death risk) or performance (ADG and $\mathrm{BW}$ ) variables among REDI and CONV in this study (Table 2). Fewer doses of antimicrobials per calf were required $(P<0.01)$ in REDI groups $(0.75 \pm 0.12)$ compared with CONV pens $(1.67 \pm 0.12)$. No calves in REDI or CONV groups died as a result of BRD without prior identification and treatment for BRD. The REDI system was used only to detect BRD and calves in each treatment group were monitored for other conditions by visual observation, and the number treated for other diseases is reported in Table 3. The REDI calves were treated earlier $(P<0.01)$ in the feeding phase relative to CONV calves (Fig. 1); $90 \%$ of the initial BRD treatments had been completed in the REDI pen at $d 16$, whereas $90 \%$ of initial BRD treatments were not completed in the CONV pen until d 22. Accordingly, days from arrival to first BRD pull were reduced in REDI calves compared with CONV cohorts $(P<0.01$; Table 2).

Mean rectal temperatures were greater $(P<0.01)$ for calves in the CONV group compared with calves in the REDI group at each identification of BRD 
Table 1. Summary of arrival characteristics and trial timelines for each of the 8 replicates variables among cattle treated with conventional management (CONV; received metaphylaxis and visual observation for bovine respiratory disease) and cattle managed using a remote early disease identification (REDI) system $^{1}$ (no metaphylaxis; bovine respiratory disease treatment decisions based on the REDI system)

\begin{tabular}{|c|c|c|c|c|c|c|c|c|c|c|}
\hline \multirow[b]{2}{*}{ Replicate } & \multirow[b]{2}{*}{ Location $^{2}$} & \multirow[b]{2}{*}{ Gender } & \multirow[b]{2}{*}{ Arrive } & \multicolumn{2}{|c|}{ CONV } & \multicolumn{2}{|c|}{ REDI } & \multicolumn{3}{|c|}{ Trial days } \\
\hline & & & & No. & $\begin{array}{c}\text { Arrival } \\
\text { weight, } \mathrm{kg}\end{array}$ & No. & $\begin{array}{c}\text { Arrival } \\
\text { weight, } \mathrm{kg}\end{array}$ & $\begin{array}{c}\text { Arrival } \\
\text { weight, SEM }\end{array}$ & $\begin{array}{l}\text { Midpoint, } \\
\text { DOF }^{3}\end{array}$ & $\begin{array}{l}\text { End, } \\
\text { DOF }\end{array}$ \\
\hline 1 & MO-1 & Heifers & May 13 & 51 & 199.2 & 52 & 196.5 & 15.5 & 33 & 59 \\
\hline 2 & MO-1 & Bulls/steers & Jul y 13 & 37 & 190.5 & 33 & 191.9 & 15.7 & 30 & 59 \\
\hline 3 & MO-1 & Heifers & Aug. 13 & 36 & 191.6 & 36 & 187.6 & 15.7 & 31 & 53 \\
\hline 4 & MO-1 & Heifers & Apr. 14 & 50 & 197.7 & 49 & 202.3 & 15.6 & 32 & 61 \\
\hline 5 & MO-1 & Bulls/steers & June 14 & 52 & 221.8 & 49 & 226.5 & 15.6 & 32 & 67 \\
\hline 6 & KS-1 & Heifers & Aug. 14 & 52 & 208.9 & 53 & 213.4 & 15.6 & 31 & -4 \\
\hline 7 & MO-2 & Heifers & Sept. 14 & 50 & 223.1 & 50 & 223.0 & 15.6 & 32 & 67 \\
\hline 8 & MO-2 & Heifers & Nov. 14 & 48 & 223.2 & 48 & 225.3 & 15.7 & 29 & 50 \\
\hline Total & & & & 376 & 207.0 & 370 & 203.0 & 10.5 & & \\
\hline
\end{tabular}

${ }^{1}$ White et al. (2015)

${ }^{2}$ MO-1 = Missouri location 1; KS-1 = Kansas location 1; MO-2 = Missouri location 2.

${ }^{3} \mathrm{DOF}=$ Days on Feed (days post-arrival).

${ }^{4}$ Replicate 6 ended prematurely at the preplanned trial midpoint of $\mathrm{d} 31$ and all data collection stopped at that point.

(Table 4). No significant interaction $(P=0.07)$ was identified between treatment group and the maximum number of BRD treatments when compared with midpoint ADG. Treatment group was not significantly associated $(P=0.70)$ with midpoint ADG, but the maximum number of treatments was significantly associated $(P<0.01)$ with calves never treated gaining more $(1.14 \pm 0.10)$ than calves treated once $(0.81 \pm$
$0.11)$, twice $(0.68 \pm 0.13)$, or 3 times $(0.33 \pm 0.15)$. Evaluation of the ADG for the entire trial period revealed a significant interaction $(P<0.01)$ among treatment group and the maximum number of times treated (Fig. 2), given that REDI calves treated twice for BRD had greater ADG $(P<0.01)$ during the trial when compared with CONV cohorts also treated twice for BRD (Fig. 2).

Table 2. Statistical comparison of health and performance variables among cattle treated with conventional management (CONV; received metaphylaxis and visual observation for bovine respiratory disease [BRD]) and cattle managed using a remote early disease identification (REDI) system ${ }^{1}$ (no metaphylaxis; BRD treatment decisions based on the REDI system $)^{2}$

\begin{tabular}{|c|c|c|c|c|}
\hline Variables evaluated & CONV & REDI & $\mathrm{SE}$ & $P$-value \\
\hline \multicolumn{5}{|l|}{ Performance } \\
\hline No., calves/group & 47.0 & 46.2 & 2.5 & 0.28 \\
\hline ADG, $\mathrm{kg}$ (mid feeding period, approximately $30 \mathrm{~d}$ ) & 0.97 & 0.89 & 0.1 & 0.23 \\
\hline ADG, kg (final, approximately $60 \mathrm{~d}$ ) & 1.09 & 1.09 & 0.1 & 1.00 \\
\hline Wt, kg (arrival) & 207.0 & 208.2 & 5.3 & 0.32 \\
\hline $\mathrm{Wt}, \mathrm{kg}$ (midpoint, approximately $30 \mathrm{~d}$ ) & 237.4 & 236.2 & 4.6 & 0.49 \\
\hline Wt, kg (final, approximately $60 \mathrm{~d}$ ) & 271.0 & 270.9 & 7.3 & 0.95 \\
\hline \multicolumn{5}{|l|}{ Health parameters } \\
\hline DOF to first BRD pull & 15.8 & 9.1 & 1.16 & $<0.01$ \\
\hline Avg doses antibiotics (per hd) & 1.67 & 0.75 & 0.12 & $<0.01$ \\
\hline \multicolumn{5}{|l|}{ Health events $\pm 95 \%$ confidence interval } \\
\hline BRD first pulls, $\%$ & $47.8 \%(34.5-61.3)$ & $51.8 \%(38.3-65.1)$ & - & 0.28 \\
\hline BRD second pulls, $\%$ & $10.6 \%(5.7-18.8)$ & $14.4 \%(8.0-24.4)$ & - & 0.10 \\
\hline BRD third pulls, $\%$ & $4.1 \%(1.8-9.1)$ & $5.1 \%(2.3-10.9)$ & - & 0.48 \\
\hline First treatment success, $\%$ & $75.9 \%(65.1-84.2)$ & $69.4 \%(57.8-79.0)$ & - & 0.16 \\
\hline Death loss, $\%$ & $4.0 \%(2.4-6.5)$ & $4.1 \%(2.5-6.6)$ & - & 0.96 \\
\hline
\end{tabular}

${ }^{1}$ White et al. (2015).

${ }^{2}$ All values represent least squares mean estimates from statistical models evaluating associations between treatment group and outcome of interest while accounting for lack of independence due to study site and replicate. 
Table 3. Descriptive statistics on treatments for medical conditions other than bovine respiratory disease (BRD) as identified by a visual observer in both cattle treated with conventional management (CONV; received metaphylaxis and visual observation for BRD) and cattle managed using a remote early disease identification (REDI) system ${ }^{1}$ (no metaphylaxis; BRD treatment decisions based on the REDI system)

\begin{tabular}{llcccc}
\hline \hline Replicate & $\begin{array}{c}\text { Treatment } \\
\text { group }\end{array}$ & Pinkeye & Lameness & $\begin{array}{c}\text { Digestive/ } \\
\text { neurological }\end{array}$ & $\begin{array}{c}\text { Total cases } \\
\text { not BRD }\end{array}$ \\
\hline 1 & CONV & 18 & 0 & 0 & 18 \\
& REDI & 5 & 0 & 0 & 5 \\
2 & CONV & 2 & 0 & 0 & 2 \\
& REDI & 1 & 3 & 1 & 5 \\
3 & CONV & 2 & 0 & 1 & 3 \\
& REDI & 0 & 1 & 2 & 3 \\
4 & CONV & 0 & 3 & 1 & 4 \\
& REDI & 4 & 0 & 0 & 4 \\
5 & CONV & 2 & 0 & 0 & 2 \\
& REDI & 1 & 3 & 0 & 4 \\
6 & CONV & 0 & 0 & 0 & 0 \\
7 & REDI & 0 & 2 & 0 & 2 \\
& CONV & 0 & 1 & 0 & 1 \\
8 & REDI & 0 & 1 & 0 & 1 \\
& CONV & 0 & 0 & 0 & 0 \\
\multirow{2}{*}{ Total } & REDI & 0 & 0 & 1 & 1 \\
& CONV & 24 & 4 & 2 & 30 \\
& REDI & 11 & 10 & 4 & 25 \\
\hline
\end{tabular}

${ }^{1}$ White et al. (2015).

\section{DISCUSSION}

High-risk beef calves were procured for the study and exhibited a substantial naturally occurring disease challenge to evaluate the $2 \mathrm{BRD}$ control systems. Key performance and health variables did not differ among groups, but potentially meaningful differences were identified among antimicrobial use and BRD treatment timing. Cattle in REDI groups required less antimicrobials and were treated earlier in the feeding phase compared with cattle in the CONV group.

One can consider the sample size relatively small ( $n=8$ pens per treatment group) and the ability to find small differences among treatment groups limited, but several performance variables (ADG and BW at multiple time points) were numerically very similar among treatment groups. The lack of difference in BW gain is surprising as the administration of metaphylaxis at arrival has been shown to increase ADG compared with not medicating calves (Schumann et al., 1991). Longerterm evaluations with increased sample size would be necessary to evaluate potential performance differences that could be related to early treatment of BRD. The number of times calves are treated for BRD has been negatively associated with ADG (Cernicchiaro

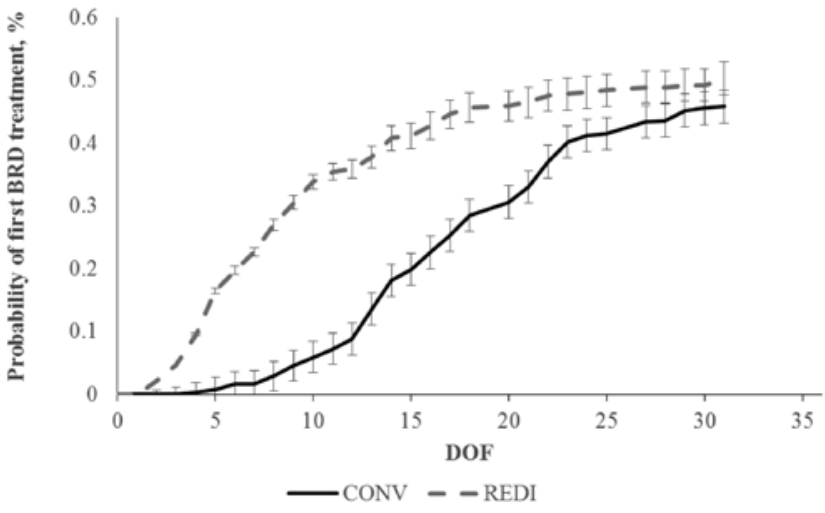

Figure 1. Survival analysis of 8 replicates comparing time to first treatment $( \pm$ SEM) among cattle treated with conventional management (CONV; grey dashed line; received metaphylaxis and visual observation for bovine respiratory disease) and cattle managed using a remote early disease identification (REDI; based on White et al. [2015]) system (black line; no metaphylaxis; bovine respiratory disease treatment decisions based on the REDI system). Cattle assigned to REDI were treated sooner $(P<0.01)$ than $\mathrm{CONV}$ cattle during the feeding phase. $\mathrm{DOF}=$ days on feed after arrival.

et al., 2013), and comparing ADG among diagnostic modalities based on the number of treatments can provide some information related to the characteristics of animals identified with each diagnostic modality. An evaluation of individual calf midpoint ADG based on treatment group and the maximum number of times treated revealed no significant interaction or treatment group effect. Similar to previous research, cattle treated a greater number of times had lower midpoint ADG compared with cattle never treated. When the entire period ADG was evaluated, a significant interaction among treatment group and the maximum number of BRD pulls was identified (Fig. 2). The interaction appears to be driven by a greater $(P<0.01)$ ADG in REDI calves treated twice compared with CONV calves treated twice. There were no total period ADG differences among REDI or CONV cattle that were never treated or were treated 1 or 3 times.

Both treatment groups faced a significant health challenge as judged by overall risk of first treatment for BRD, yet statistical differences were not identified between risk of first treatment, first treatment success, and death loss risk among the 2 BRD management schemes (Table 2). This was surprising as metaphylaxis has been reported to reduce expected morbidity by up to $50 \%$ (Nickell and White, 2010), but the REDI group had no difference in morbidity risk compared with the CONV group, which received metaphylaxis. A potential explanation for these results is that the REDI system accurately identified BRD cases early in the disease process, which may have further limited the disease outbreak in the pen.

Diagnosis of BRD is commonly based on visual assessment of animal attitude, appetite, respiratory pattern, and social interactions (Lechtenberg et al., 
Table 4. Statistical comparison of rectal temperature at each identification (first, second, or third treatment) for bovine respiratory disease (BRD) among cattle treated with conventional management (CONV; received metaphylaxis and visual observation for BRD) and cattle managed using a remote early disease identification (REDI) system ${ }^{1}$ (no metaphylaxis; BRD treatment decisions based on the REDI system $)^{2}$

\begin{tabular}{llllc}
\hline \hline \multirow{2}{*}{ BRD treatment } & \multicolumn{4}{c}{ Rectal temperature } \\
\cline { 2 - 5 } & CONV & REDI & SE & $P$-value \\
\hline First & 40.6 & 40.3 & 0.08 & $<0.01$ \\
Second & 40.5 & 39.8 & 0.15 & $<0.01$ \\
Third & 40.6 & 40.0 & 0.20 & $<0.01$ \\
\hline
\end{tabular}

${ }^{1}$ White et al. (2015).

${ }^{2}$ All values represent least squares mean estimates from statistical models evaluating associations between treatment group and outcome of interest while accounting for lack of independence due to study site and replicate.

1998; Smith et al., 2001). None of the clinical signs associated with BRD are pathognomic, and previous studies (Wittum et al., 1996; Schneider et al., 2009; Amrine et al., 2013) demonstrated the relatively low diagnostic accuracy of using visual observations when compared with lung lesions. Rectal temperature at the time of treatment is often used to augment the therapeutic decision (Perino and Apley, 1998), and rectal temperature was a part of the case definition in the CONV group but not in the REDI group. Therefore, it is not surprising that the rectal temperature at first treatment for BRD was higher in the CONV group as rectal temperature greater than $40^{\circ} \mathrm{C}$ was part of the case definition for treatment, which limited the range and likely influenced the variability of temperatures in the CONV group. Although a statistical difference was identified, both CONV and REDI had mean rectal temperature at first BRD treatment of greater than $40^{\circ} \mathrm{C}$, a commonly used threshold to augment BRD diagnosis. Even when combined with visual assessment, rectal temperature is not a perfect prognostic indicator of case outcome (Theurer et al., 2014).

The REDI system has been shown to identify calves up to $0.75 \mathrm{~d}$ earlier than a trained observer (White et al., 2015), and these previous results are circumstantially supported by the fact that calves in the REDI group were treated earlier in the feeding phase. Calves in the REDI group in the current study may have been identified earlier in the feeding phase compared with previous work due to concurrent administration of metaphylaxis in the CONV group. The diagnosis with the REDI system is based on changes in behavior and previous research has illustrated that temperament is associated with the risk for BRD (Buczinski et al., 2015). The earlier treatments in the REDI pens could

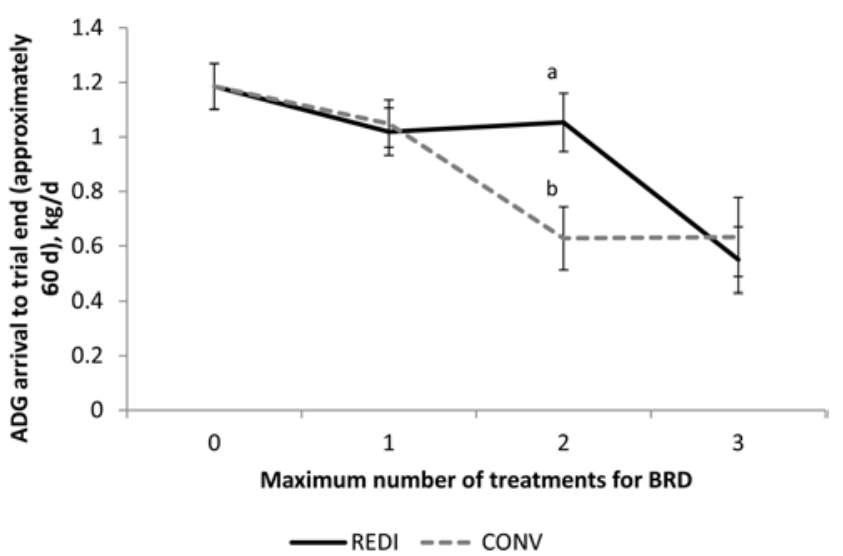

Figure 2. Results of generalized linear model identifying significant $(P<$ $0.01)$ interaction among treatment group and the maximum times treated for bovine respiratory disease (BRD) during the period with $\mathrm{ADG}$ over the trial period. Data represent least squares mean $( \pm \mathrm{SE})$ period $\mathrm{ADG}$ comparing cattle treated with conventional management (CONV; grey dashed line; received metaphylaxis and visual observation for BRD) and cattle managed using a remote early disease identification (REDI; based on White et al. [2015]) system (black line; no metaphylaxis; BRD treatment decisions based on the REDI system) based on the maximum number of BRD treatments during the study. Least squares means with unique superscripts differ statistically $(P<0.01)$.

be influenced by the 5-d postmetaphylaxis interval used in the CONV pens; however, Fig. 1 illustrates that very few calves were identified as BRD positive in the CONV pens prior to $\mathrm{d} 10$. The difference in treatment timing could also result from the impact of metaphylaxis modifying bacterial populations in the CONV calves resulting in later occurrence of BRD. Accordingly, diagnostic accuracy cannot be directly compared in this research as the diagnostic modalities (visual observation or REDI) are completely confounded by the administration of metaphylaxis to the CONV group. However, both treatment groups exhibited no differences in initial morbidity risk or posttreatment outcomes (first treatment success and mortality risk). Further research comparing known pulmonary outcomes is needed to evaluate if potential differences exist in diagnostic accuracy of these modalities, which may explain the findings of the current research.

In addition to potentially limiting disease outbreaks, early treatment may have advantages including improved performance (Babcock et al., 2009) and the implications on labor management. If the majority of BRD treatments occur in a short, well-defined period (e.g., the first $2 \mathrm{wk}$ after arrival), available resources can be efficiently concentrated to manage calves during this focused time period. The high risk period for initial BRD cases is often considered to be the first $45 \mathrm{~d}$ after arrival to the feed yard (Babcock et al., 2010). Previous authors have suggested that one of the reasons for high morbidity risk at specific times of year could be related to a relatively high number of cattle to be monitored within a short period of time that can overwhelm the 
labor force (Ribble et al., 1995). A system that could reduce the high-risk period from 45 to $15 \mathrm{~d}$ would be valuable to commercial feeding operations as the labor management plan could be optimized allowing focus on high-risk pens yet not inhibiting the incoming cattle flow. One limitation of the REDI system is it was used only to identify BRD cases and pen riders were still required to monitor for other disease conditions.

Given the lack of health risk and performance differences, one of the more remarkable findings is related to the average total antimicrobial use per calf. This difference appears to due to the fact that REDI calves received antimicrobials only at the time of disease identification and not as a mass population treatment at arrival. If the diagnostic modality is accurate, administering antimicrobial therapy only at the time of disease identification represents rational use of antimicrobials, which has been cited as an important strategy to control antimicrobial resistance (Centers for Disease Control and Prevention, 2013). Reducing the total antimicrobials used offers several other potential benefits including decreased expenses and public perception regarding evidence-based antimicrobial use.

In aggregate, these findings illustrate that REDI provided no differences in performance to conventional management of visual observation and metaphylaxis but reduced antimicrobial use and days to first treatment. Further research needs to be performed to fully evaluate potential differences among the systems in varied environments.

\section{LITERATURE CITED}

Amrine, D. E., B. J. White, R. L. Larson, D. E. Anderson, D. A. Mosier, and N. Cernicchiaro. 2013. Determining precision and accuracy of clinical illness scores compared to pulmonary consolidation scores in Holstein calves with induced Mycoplasma bovis pneumonia. Am. J. Vet. Res. 74(2):310 315. doi:10.2460/ajvr.74.2.310.

Babcock, A. H., D. G. Renter, B. J. White, and S. R. Dubnicka. 2010. Temporal distributions of respiratory disease events within cohorts of feedlot cattle and associations with cattle health and performance indices. Prev. Vet. Med. 97(34):198-219. doi:10.1016/j.prevetmed.2010.09.003.

Babcock, A. H., B. J. White, S. S. Dritz, D. U. Thomson, and D. G. Renter. 2009. Feedlot health and performance effects associated with the timing of respiratory disease treatment. J. Anim. Sci. 87:314-327. doi:10.2527/jas.2008-1201.

Brooks, K. R., K. C. Raper, C. E. Ward, B. P. Holland, C. R. Krehbiel, and D. L. Step. 2011. Economic effects of bovine respiratory disease on feedlot cattle during backgrounding and finishing phases. Prof. Anim. Sci. 27:195-203.

Buczinski, S., R. D. Rademacher, H. M. Tripp, M. Edmonds, E. G. Johnson, and S. Dufour. 2015. Assessment of 1-lactatemia as a predictor of respiratory disease recognition and severity in feedlot steers. Prev. Vet. Med. 118:306-318. doi:10.1016/j. prevetmed.2014.12.003.
Centers for Disease Control and Prevention. 2013. Antibiotic resistance threats in the United States, 2013. http://www.cdc.gov/drugresistance/threat-report-2013/index.html. Accessed May 15, 2015.

Cernicchiaro, N., B. J. White, D. G. Renter, and A. H. Babcock. 2013. Evaluation of economic and performance outcomes associated with the number of treatments after an initial diagnosis of bovine respiratory disease in commercial feeder cattle. Am. J. Vet. Res. 74:300-309. doi:10.2460/ajvr.74.2.300.

Fulton, R. W., B. J. Cook, D. L. Step, A. W. Confer, J. T. Saliki, M. E. Payton, L. J. Burge, R. D. Welsh, and K. S. Blood. 2002. Evaluation of health status of calves and the impact on feedlot performance: Assessment of a retained ownership program for postweaning calves. Can. J. Vet. Res. 66:173-180.

Hanzlicek, G., B. White, D. Mosier, D. Renter, and D. Anderson. 2010. Serial evaluation of physiologic, pathological, and behavioral changes related to disease progression of experimentally induced Mannheimia haemolytica pneumonia in postweaned calves. Am. J. Vet. Res. 71:359-369. doi:10.2460/ ajvr.71.3.359.

Lechtenberg, K. F., R. A. Smith, and G. L. Stokka. 1998. Feedlot health and management. Vet. Clin. North Am. Food Anim. Pract. 14:177-197.

Nickell, J., and B. J. White. 2010. Metaphylactic antimicrobial therapy for bovine respiratory disease in stocker and feedlot cattle. Vet. Clin. North Am. Food Anim. Pract. 26:285-301. doi:10.1016/j.cvfa.2010.04.006.

Perino, L. J., and M. Apley. 1998. Clinical trial design in feedlots. Vet. Clin. North Am. Food Anim. Pract. 14:243-266.

Ribble, C. S., A. H. Meek, E. D. Janzen, P. T. Guichon, and G. K. Jim. 1995. Effect of time of year, weather, and the pattern of auction market sales on fatal fibrinous pneumonia (shipping fever) in calves in a large feedlot in Alberta (1985-1988). Can. J. Vet. Res. 59:167-172.

Schneider, M. J., R. G. Tait Jr., W. D. Busby, and J. M. Reecy. 2009. An evaluation of bovine respiratory disease complex in feedlot cattle: Impact on performance and carcass traits using treatment records and lung lesion scores. J. Anim. Sci. 87:1821-1827. doi:10.2527/jas.2008-1283.

Schumann, F. J., E. D. Janzen, and J. J. McKinnon. 1991. Prophylactic medication of feedlot calves with tilmicosin. Vet. Rec. 128:278-280. doi:10.1136/vr.128.12.278.

Smith, R. A. 2009. North American cattle marketing and bovine respiratory disease (BRD). Anim. Health Res. Rev. 10:105108. doi:10.1017/S1466252309990107.

Smith, R. A., G. L. Stokka, O. Radostits, and D. Griffin. 2001. Health and production management in beef feedlots. In: O. Radostits, editor, Herd health: Food animal production medicine. WB Saunders Company, Philadelphia, PA. p. 592-595.

Theurer, M. E., D. E. Amrine, and B. J. White. 2013a. Remote noninvasive assessment of pain and health status in cattle. Vet. Clin. North Am. Food Anim. Pract. 29:59-74. doi:10.1016/j.cvfa.2012.11.011.

Theurer, M. E., D. E. Anderson, B. J. White, M. D. Miesner, D. A. Mosier, J. F. Coetzee, J. Lakritz, and D. E. Amrine. 2013b. Effect of Mannheimia haemolytica pneumonia on behavior and physiologic responses of calves during high ambient environmental temperatures. J. Anim. Sci. 91:3917-3929. doi:10.2527/jas.2012-5823.

Theurer, M. E., B. J. White, R. L. Larson, K. K. Holstein, and D. E. Amrine. 2014. Relationship between rectal temperature at first treatment for bovine respiratory disease complex in feedlot calves and the probability of not finishing the production cycle. J. Am. Vet. Med. Assoc. 245:1279-1285. doi:10.2460/javma.245.11.1279. 
USDA. 2013. Feedlot 2011 part I: Management practices on U.S. feedlots with a capacity of 1,000 or more head. No. 626.0313 . USDA-APHIS-VS, CEAH., Fort Collins, CO.

White, B. J., D. E. Anderson, D. G. Renter, R. L. Larson, D. A. Mosier, L. L. Kelly, M. E. Teurer, B. D. Robert, and M. L. Walz. 2012. Clinical, behavioral, and pulmonary changes in calves following inoculation with Mycoplasma bovis. Am. J. Vet. Res. 73:490-497. doi:10.2460/ajvr.73.4.490.

White, B. J., D. R. Goehl, and D. E. Amrine. 2015. Comparison of a remote early disease identification (REDI) system to visual observations to identify cattle with bovine respiratory diseases. Int. J. Appl. Res. Vet. Med. 13:23-30.

White, B. J., and D. G. Renter. 2009. Bayesian estimation of the performance of using clinical observations and harvest lung lesions for diagnosing bovine respiratory disease in post-weaned beef calves. J. Vet. Diagn. Invest. 21:446-453. doi:10.1177/104063870902100405.
Wittum, T. E., N. E. Woollen, L. J. Perino, and E. T. Littledike. 1996. Relationships among treatment for respiratory tract disease, pulmonary lesions evident at slaughter, and rate of weight gain in feedlot cattle. J. Am. Vet. Med. Assoc. 209:814-818.

Wolfger, B., B. J. Manns, H. W. Barkema, K. S. SchwartzkopfGenswein, C. Dorin, and K. Orsel. 2015a. Evaluating the cost implications of a radio frequency identification feeding system for early detection of bovine respiratory disease in feedlot cattle. Prev. Vet. Med. 118:285-292. doi:10.1016/j. prevetmed.2014.12.001.

Wolfger, B., K. S. Schwartzkopf-Genswein, H. W. Barkema, E. A. Pajor, M. Levy, and K. Orsel. 2015b. Feeding behavior as an early predictor of bovine respiratory disease in North American feedlot systems. J. Anim. Sci. 93:377-385. doi:10.2527/jas.2013-8030. 
Reproduced with permission of the copyright owner. Further reproduction prohibited without permission. 